\title{
Les pharmaciens, gardiens de l'utilisation sécuritaire des médicaments
}

\author{
par Régis Vaillancourt
}

$\mathrm{D}$ ans ce numéro du JCPH, Al Hamarneh et ses collègues ${ }^{1}$ font état d'une étude intéressante dans laquelle ils ont évalué les perceptions qu'ont les pharmaciens d'hôpitaux de leur propre rôle. Dans cette étude, on a notamment demandé aux pharmaciens d'hôpitaux de l'Alberta de décrire leurs fonctions. La majorité des réponses concernaient des activités consacrées aux médicaments ou à la distribution des médicaments; seule une minorité de réponses portaient sur des activités axées sur les patients. Les auteurs ont conclu que les pharmaciens d'hôpitaux n'avaient peut-être pas complètement adhéré au concept des soins axés sur le patient tel que décrit dans le Plan directeur pour la pharmacie ${ }^{2}$.

Je crois qu'il est important que les pharmaciens d'hôpitaux concentrent leur pratique sur les soins aux patients. Cependant, j'argumenterais que la perception qu'ont certains pharmaciens d'hôpitaux de leur rôle principalement à titre de distributeurs de médicaments, comme l'ont observé $\mathrm{Al}$ Hamarneh et ses collègues, est en fait conforme aux exigences essentielles de la prestation des soins pharmaceutiques. Ce sentiment est d'ailleurs traduit dans la vision du Plan directeur pour la pharmacie $^{2}$ de même que dans les énoncés consensuels de Bâle sur la pharmacie hospitalière publiés par la Fédération internationale pharmaceutique (FIP) ${ }^{3}$.

En 1992, Strand et ses collaborateurs ${ }^{4}$ ont affirmé que les services de pharmacie nécessaires à la prestation des soins pharmaceutiques doivent inclure la distribution des médicaments de façon précise et en temps opportun, les données sur les patients précises et disponibles en temps opportun, l'information sur les médicaments complète et actuelle ainsi que la consignation des décisions et des interventions des pharmaciens. Dix-huit ans plus tard, je crois que ces services de pharmacie restent des éléments fondamentaux de la pratique. En fait, les avantages liés à la prestation de services de pharmacie clinique évolués sont perdus si l'élément " distribution des médicaments de façon précise et en temps opportun » est absent et que le patient ne reçoit pas les bons médicaments. Ce point de vue a été validé plus récemment dans une campagne de valorisation de la $\mathrm{SCPH}^{5}$ et une allocution au congrès annuel de 2007 de l'American College of Clinical Pharmacy (ACCP) ${ }^{6}$.
La campagne de valorisation de la SCPH en juin 2008 gravitait autour de la distribution optimale des médicaments et de son incidence sur la sécurité des patients. L'un des messages clés de la campagne était que « Pour assurer la sécurité des soins prodigués aux patients, il est essentiel que des systèmes de distribution des médicaments sûrs, précis, efficients et bien conçus soient mis en place. " ${ }^{5}$ Que la distribution des médicaments soit comprise dans le rôle autoperçu du pharmacien d'hôpital n'exclut ni ne contredit le concept des soins axés sur le patient. Cet argument a aussi été repris par Koda-Kimble au congrès annuel de 2007 de l'ACCP. Koda-Kimble a souligné que dans la foulée de nos efforts à élargir notre champ de pratique aux soins axés sur le patient, nous devons continuer d'assumer notre responsabilité fondamentale de gardiens des systèmes de gestion des médicaments et de l'assurance continue de la qualité ${ }^{6}$. Elle a donné l'exemple d'un établissement où les pharmaciens cliniciens et les pharmaciens assurant la distribution des médicaments travaillaient en vase clos, ce qui a abouti à des pratiques de gestion des médicaments inacceptables et à une diminution globale de l'intégrité du système de distribution des médicaments ${ }^{6}$.

Lénoncé de vision du Plan directeur pour la pharmacie " procurer aux Canadiens un traitement médicamenteux aux résultats optimaux par des soins axés sur le patient $"^{2}$ — est sans l'ombre d'un doute centré sur les patients. En revanche, ce plan ne propose pas que la profession de pharmacien néglige pour autant ses responsabilités en matière de distribution des médicaments. En fait, le plan stipule que les pharmaciens et les techniciens en pharmacie ont un double mandat, soit d'assurer la sécurité et l'intégrité du système de distribution des médicaments et également de diriger l'élaboration de mesures visant à améliorer la sécurité et la qualité des médicaments et de contribuer à ces mesures ${ }^{2}$. Létude menée par $\mathrm{Al} \mathrm{Hamarneh} \mathrm{et} \mathrm{ses}$ collaborateurs montre que les pharmaciens d'hôpitaux définissent leur rôle au moyen d'un élément essentiel des soins : la bonne dose du bon médicament, administrée au bon patient, au bon moment et par la bonne voie - en d'autres mots, le système de distribution des médicaments. 
Les énoncés consensuels de Bâle de la FIP sur la pratique de la pharmacie hospitalière ${ }^{3}$ appuient l'élargissement des activités de soins aux patients prodigués par les pharmaciens, mais ils soutiennent également le rôle des pharmaciens en tant que gardiens du système de gestion des médicaments. En fait, certains des énoncés consensuels appuient directement la prise en charge du système de distribution des médicaments par les pharmaciens : "Les autorités de santé et directeurs des hôpitaux devraient impliquer les pharmaciens hospitaliers dans toutes les étapes du processus d'utilisation du médicament à l'hôpital " et "Les pharmaciens hospitaliers devraient prendre une responsabilité dans tous les circuits logistiques des médicaments à l'hôpital $»^{3}$. Ces énoncés définissent clairement le rôle des pharmaciens d'hôpitaux en regard du système de distribution des médicaments, en plus de leurs activités cliniques.

Les exigences relatives à la pratique des soins pharmaceutiques, le Plan directeur pour la pharmacie et les énoncés consensuels de Bâle de la FIP appuient tous le rôle des pharmaciens dans la gestion de systèmes efficaces de distribution des médicaments favorisant des soins sécuritaires aux patients. Bien que la distribution des médicaments ne doive pas être l'apanage de la pratique de la pharmacie hospitalière, elle en constitue néanmoins un élément nécessaire qui ne saurait exclure ou remplacer la prestation des soins axés sur le patient. Malgré que les pharmaciens d'hôpitaux doivent s'impliquer davantage dans la prestation des soins aux patients, ils ne doivent pas oublier leur rôle comme gardiens des systèmes de gestion des médicaments dans la foulée de l'élargissement de leur champ de pratique.

[Traduction par l'éditeur]
Références

1. Al Hamarneh YN, Rosenthal M, McElnay JC, Tsuyuki RT. Pharmacists' perceptions of their professional role: insights into hospital pharmacy culture. Can J Hosp Pharm 2011;64(1):31-35.

2. Plan directeur pour la pharmacie: Concevoir l'avenir ensemble. Ottawa $(\mathrm{ON})$ : Association des pharmaciens du Canada; 2009. Publié au www. pharmacists.ca/content/about_cpha/whats_happening/cpha_in_action/pdf/ BlueprintImplementationPlan_FR.pdf. Consulté le 17 décembre 2010.

3. FIP Global Conference on the Future of Hospital Pharmacy: final Basel statements. La Haye (Pays-Bas) : International Pharmaceutical Federation (FIP); 4 décembre 2008. Publié au http://www.fip.org/files/fip/ HPS/Basel2008/translations/BaselStatementsFrench.pdf. Consulté le 30 novembre 2010.

4. Strand LM, Cipolle RJ, Morley PC. Pharmaceutical care: an introduction. Kalamazoo (MI): The Upjohn Company; 1992.

5. Medication safety enhanced by drug distribution - 2008 [campagne de valorisation]. Ottawa (ON): Canadian Society of Hospital Pharmacists; 2008. Publié au www.cshp.ca/advocacy/campaigns/medSafety_e.asp. Consulté le 30 novembre 2010

6. Koda-Kimble issues challenge. Parker Medal winner calls for action through contributions to ACCP Research Institute. ACCP Rep 2007;26(11):8-9.

Régis Vaillancourt, O.M.M., C.D., B. Pharm., Pharm. D., FCSHP, est directeur de pharmacie au Centre hospitalier pour enfants de l'est de I'Ontario, à Ottawa, en Ontario. II est aussi rédacteur adjoint au JCPH.

Adresse de correspondance :

Dr Régis Vaillancourt

Centre hospitalier pour enfants de l'est de l'Ontario

401, chemin Smyth

Ottawa (ON) K1H 8L1

Courriel : RVaillancourt@cheo.on.ca

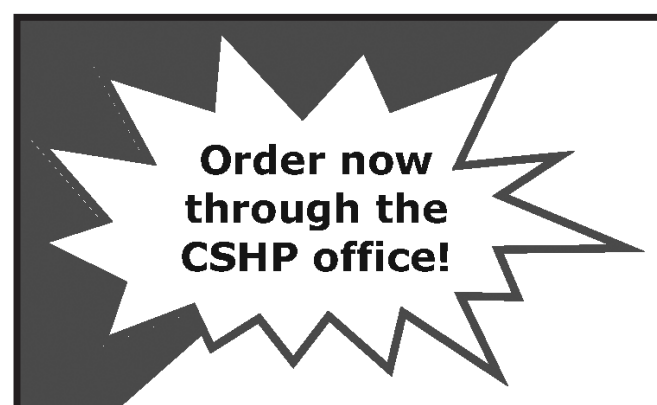

\section{Safe and Effective}

The Eight Essential Elements of an Optimal Medication-Use System

Editor: Neil J. MacKinnon, PhD, FCSHP

Medication is the most relied-upon treatment in health care today. Despite its importance, the current medication-use system suffers from problems related to lack of safety and quality. Safe and Effective explores the medication-use system and, more importantly, provides concrete and straightforward strategies for improving it.

"The patient safety movement is profoundly changing the face of modern health care. This effort by Neil Mackinnon and his capable and progressive group of colleagues is a "must read" for clinicians, practitioners, academics and students who are part of this important journey. This addition to the literature will assist in advancing patient safety practices for Canadian caregivers and will most certainly benefit those we all serve." - Phil Hassen, CEO, Canadian Patient Safety Institute

Now available to order through the CSHP office - $\$ 81$ for CSHP members and $\$ 90$ for nonmembers (plus applicable taxes and shipping). Please find our order form online at www.cshp.ca under the "Products and Publications" drop-down menu. 